\title{
Relato de experiência de uma oficina sobre sexualidade com jovens de uma escola pública de Uberlândia, Minas Gerais
}

\author{
Experience report of a workshop on youth sexuality at a public school in Uberlândia, State of \\ Minas Gerais, Brazil
}

\author{
Mariana Vasconcelos Paranaiba ${ }^{1}$ \\ Gabriela Ferreira de Camargos Rosa ${ }^{2}$ \\ Mariana Hasse ${ }^{3}$ \\ Elisa Toffoli Rodrigues ${ }^{4}$
}

\section{RESUMO}

De acordo com as Diretrizes Curriculares Nacionais para o curso de Medicina, compete aos estudantes de medicina conhecer, vivenciar e efetivar as políticas públicas de saúde. Diante disso, nós, três estudantes de medicina da Universidade Federal de Uberlândia (UFU) e 2 coordenadoras, docentes do curso, elaboramos um projeto de extensão, com início em março de 2018 e término em janeiro de 2019. O objetivo foi abordar o conceito de redução de danos, através da educação popular em saúde, em uma escola pública da cidade. Iniciamos o projeto com a pactuação das atividades com a escola escolhida. Optamos por uma escola situada na área central da cidade que oferecia ensino médio noturno, na modalidade regular e também do programa de Educação para Jovens e Adultos. Dentre os diversos temas sugeridos o tópico sexualidade apareceu na maioria dos votos. Foi desenvolvida uma oficina sobre sexualidade em quatro etapas: aquecimento, atividade 1, atividade 2 e feedback. Participaram da oficina 52 estudantes de três turmas diferentes, uma de cada ano do Ensino Médio. Conseguimos, com dificuldade, envolver a maior parte dos participantes, permitindo a participação e exposição de ideias diversas, surgimento de polêmicas e informações enriquecedoras a todo o grupo.

Palavras-chave: Sexualidade. Educação da população. Redução de danos.

\begin{abstract}
According to the National Curriculum Guidelines for the Medical course, it is up to medical students to know, experience and implement public health policies. Considering this, we, 3 medical students from the Federal University of Uberlândia (UFU) and 2 coordinators, professors of the UFU medical course, prepared an extension project, beginning in March 2018 and ending in January 2019, with the objective of bringing the concept of Harm Reduction to the reality of young people from a public school in the city through Popular Health Education. We started the project with the agreement for the activities by the chosen school. We opted for a school located in the central area of the city that offered evening high school, in regular modalities and also the program of Education for Youth and Adults. Among

\footnotetext{
1 Graduanda em Medicina na Universidade Federal de Uberlândia, Minas Gerais, Brasil (marianaparanaiba@hotmail.com).

2 Graduada em Medicina pela Universidade Federal de Uberlândia, Minas Gerais, Brasil (gabrielacamargosrosa@outlook.com).

3 Doutora em Enfermagem pela Universidade de São Paulo, Brasil; professora adjunta da Faculdade de Medicina da Universidade Federal de Uberlândia, Minas Gerais, Brasil (marianahasserp@ gmail.com).

${ }^{4}$ Doutoranda em Saúde Coletiva na Universidade Estadual de Campinas, São Paulo, Brasil; professora da Faculdade de Medicina da Universidade Federal de Uberlândia, Minas Gerais, Brasil; (elisa_toffoli@yahoo.com.br).
} 
the various suggested topics, the topic sexuality appeared in most votes. The workshop on sexuality was then built in four stages, namely: warm-up, activity 1, activity 2 and space for feedback. A total of 52 students from three different classes, one from each year of high school, participated in the workshop. However, with difficulty, we were able to involve most students in the classroom, allowing the participation and exposure of diverse ideas, the emergence of controversy and enriching information to the whole group.

Keywords: Sexuality. Population education. Harm reduction.

\section{INTRODUÇÃO}

De acordo com as Diretrizes Curriculares Nacionais (DCN) para o Curso de Medicina (BRASIL, 2014) compete aos graduandos conhecer, vivenciar e efetivar as políticas públicas de saúde. Para isso, entre outras atividades, devem desenvolver ações de promoção da saúde que respondam às necessidades da sociedade.

Dentre as políticas discutidas e trabalhadas com os estudantes de medicina na Universidade Federal de Uberlândia (UFU) está a Política para Atenção Integral a Usuários de Álcool e Outras Drogas (BRASIL, 2004) que propõe a estratégia de Redução de Danos.

Nesse contexto, a Redução de Danos estabelece ações destinadas aos usuários de drogas, lícitas e ilícitas, com o objetivo de reduzir os riscos associados ao uso e promover a saúde de modo abrangente, considerando a autonomia dos sujeitos. Dentre as diferentes ações propostas, ressalta-se a orientação para a prática do sexo seguro e a prevenção de infecções como HIV e hepatites (BRASIL, 2004), informando e empoderando os sujeitos acerca de sua saúde sexual e reprodutiva.

Sabe-se que, apesar da disponibilidade de uma infinidade de informações acerca das Infecções Sexualmente Transmissíveis (IST) e da prevenção de gravidez indesejada, ainda é bastante frequente suas ocorrências entre os adolescentes e jovens, o que se constitui como um problema de saúde pública no nosso país.

Em consonância com as DCN, a Política Nacional de Educação Popular em Saúde (PNEPSSUS) (BRASIL, 2013a) - também abordada durante a graduação em Medicina - estimula os estudantes a produzirem ações de promoção em saúde e prevenção de doenças voltadas à comunidade, buscando, através de informação e construção de diálogo, a autonomia e empoderamento das pessoas no que tange às decisões que afetam sua própria saúde (RAIMONDI et al., 2018). 
Assim, as Políticas de Redução de Danos e de Educação Popular em Saúde apresentam objetivos e princípios que as aproximam e complementam. Ambas propõem que os indivíduos atuem como protagonistas de suas vidas, a partir da informação adequada, problematização da realidade e construção de estratégias conjuntas para promoção de saúde.

Tais propostas alinham-se ao conceito da salutogênese criado por Aaron Antonovsk a partir de estudos com mulheres submetidas aos campos de concentração nazista. A partir de tais estudos, ele notou que, apesar de submetidas a situações semelhantes de estresse físico e psicológico, algumas mulheres se mantinham saudáveis enquanto outras adoeciam. $\mathrm{O}$ conceito de salutogênese (do latim: salus = saúde; e do grego: genesis = origem), um dos mais desenvolvidos e trabalhados no âmbito da promoção da saúde atualmente, representou uma mudança de paradigma nas ciências da saúde. Isso porque ele busca identificar as razões que levam alguém a estar saudável, ou seja, procura por recursos protetivos que auxiliam as pessoas a alcançarem saúde (LINDSTRÖM, 2018).

Segundo tal conceito, a promoção de saúde ocorre por meio do desenvolvimento do sentido de coerência de cada indivíduo, que é formado pela: 1) capacidade de compreensão (maneira que o indivíduo apreende estímulos e informações); 2) capacidade de gestão (percepção do indivíduo de seus recursos pessoais e sociais) e 3) capacidade de investimento (capacidade de investir os recursos que possui na criação de mecanismos para superar as situações adversas) (LINDSTRÖM, 2018; SALCI et al., 2013).

Assim, através do sentido de coerência, os indivíduos são capazes de compreender melhor sua saúde e as variáveis que nela interferem, a partir de uma reflexão crítica e ampliada de suas individualidades e do coletivo onde está inserido, a fim de potencializar sua capacidade de gestão sobre si e produção de investimentos em comportamentos e ações que produzam saúde (ABREU; ALONZO, 2018; LINDSTRÖM, 2018; SALCI et al., 2013).

Dessa forma, a salutogênese na promoção de saúde alinha-se com o conceito de saúde proposto pela Organização Mundial de Saúde (OMS), em meados de 1950, que amplia o entendimento de saúde para além da ausência de doença, propondo uma abordagem da saúde como um estado positivo, como um recurso para viver a vida em sua plenitude. Nesse contexto, entende-se que o processo de saúde-adoecimento-cuidado é influenciado por diversos fatores internos e externos aos indivíduos, que precisam ser reconhecidos e trabalhados para que as ações de Educação Popular em Saúde sejam potencializadas e tornem- 
se, de fato, resolutivas e empoderadoras (LINDSTRÖM, 2018; FRAGA et al., 2013; SALCI et al., 2013). A partir disso, a saúde pode ser efetivamente produzida a partir de uma abordagem holística, empoderando indivíduos e comunidades, promovendo a autonomia para a saúde pública, ações intersetoriais para construir políticas públicas em todos os setores e criando sistemas de saúde sustentáveis (KUMAR; PREETHA, 2012).

Para isso, as ações em saúde não podem se restringir à imposição de comportamentos e ideais, dissociados da realidade e cotidiano dos indivíduos. Pelo contrário devem ser um meio para promoção da participação em intervenções na realidade concreta da vida de cada sujeito, almejando a qualidade de vida, ou seja, ser uma educação para a cidadania (PEDROSA, 2006).

Assim, elaboramos um projeto de extensão com o objetivo de desenvolver atividades baseadas nos princípios de Redução de Danos com jovens de uma escola pública da cidade, a partir da Educação Popular em Saúde, pautada na compreensão da salutogênese. Apresentaremos a seguir nossa experiência com uma das oficinas realizadas durante o projeto, quando trabalhamos a temática da sexualidade.

\section{METODOLOGIA}

Nós, três estudantes da faculdade de medicina da UFU e 2 coordenadoras, docentes também da instituição, iniciamos o projeto com a pactuação das atividades com a escola escolhida. Optamos por uma escola situada na área central da cidade que oferecia ensino médio noturno, nas modalidades regular e, também, no programa de Educação para Jovens e Adultos (EJA). Assim, trabalhamos com estudantes entre 16 e 25 anos, entre março de 2018 e janeiro de 2019.

Foi pactuado com a direção da escola parte da carga horária curricular dos estudantes para a realização de oficinas sobre diversos temas, utilizando princípios da Redução de Danos e da Educação Popular em Saúde através de metodologias participativas.

Inicialmente, realizamos um diagnóstico situacional do ambiente escolar para identificar informantes chaves e as principais vulnerabilidades daquele contexto. Para isso, realizamos visitas diárias à escola por um mês, nas quais interagíamos com professores, funcionários e estudantes, e buscávamos informações em registros oficiais da escola (cadernos de 
ocorrências e livros de atas). Nesse momento, disponibilizamos urnas para que todos sugerissem temas para as atividades do projeto.

Dentre diversos temas sugeridos, como futuro profissional, uso de drogas, política e religião, o tema da sexualidade foi prioritário entre os jovens e abrangeu questões relacionadas a planejamento familiar, infecções sexualmente transmissíveis (IST) e aborto.

Considerando os princípios propostos pela PNEPS-SUS, buscamos materiais que nos auxiliassem a trabalhar o tema da sexualidade com os jovens a partir de metodologias participativas.

A oficina foi construída para ser realizada em quatro etapas: aquecimento, atividade 1, atividade 2 e espaço para ofeedback.

O aquecimento, chamado de "Como vai você?" foi adaptado do livro Oficina de ideias Manual de dinâmicas (CROMACK, 2003), e consistiu em convidar os participantes a andarem pela sala, observando uns aos outros, enquanto tocava uma música. Ao pausarmos a música, eles formavam duplas com a pessoa mais próxima e se apresentavam.

As duas atividades seguintes foram adaptadas do livro Saúde e prevenção nas escolas: guia para a formação de profissionais de saúde e de educação do Ministério da Saúde (BRASIL, 2006). Na primeira, denominada "Concordo ou não concordo?", foram colados dois cartazes, um escrito "concordo" e outro escrito "não concordo", em lados opostos da sala. A partir disso, foram lidas afirmações referentes à temática da sexualidade e, após a leitura, os estudantes se posicionavam próximo ao cartaz que melhor expressava a sua opinião. Posteriormente, fazíamos breves questionamentos e comentários sobre cada afirmativa.

Na segunda atividade, chamada "E agora, o que fazer?", dividimos os estudantes em seis grupos, cada um com aproximadamente oito estudantes e distribuímos duas situações problemas, sendo elas: 1) 1) Um casal de namorados usava camisinha como contracepção e prevenção de IST, porém o rapaz passou a se recusar a usar e falou para a namorada tomar pílula, por ser um método mais fácil. A moça nunca havia tomado pílula e não desejava utilizá-la, pois temia os efeitos colaterais. No momento, mantém relações sexuais; 2) Uma adolescente acaba de ser aprovada na faculdade e vai à sua primeira festa universitária. $\mathrm{Na}$ festa encontra um veterano do seu curso que lhe parece interessante. Eles vão até um dos quartos da casa e começam a se beijar. Ela sentia desejo e curiosidade, porém não estava 
segura e não desejava ir além dos beijos. Ele, por sua vez, a todo momento tentava tocar em todo o seu corpo e tirar suas roupas.

Os estudantes discutiram os casos problematizando as situações apresentadas e pensando em possíveis soluções, as quais foram posteriormente compartilhadas com o restante da turma. Durante a discussão, nós circulávamos entre os pequenos grupos, atuando como mediadores, acompanhando as discussões e fazendo questionamentos.

Ao final disponibilizamos um espaço aberto para que os estudantes expressassem suas opiniões sobre a oficina e sugestões de aprimoramento. $\mathrm{O}$ tempo utilizado para a atividade foi de 90 minutos.

\section{RESULTADOS E DISCUSSÃO}

Participaram da oficina um total de 52 estudantes de três turmas diferentes, uma de cada ano do Ensino Médio. Ao nos reunirmos em um mesmo espaço físico, ficou nítida a divisão entre as turmas, bem como a dificuldade que teríamos para que se organizassem e fizessem silêncio para o início da atividade. Explicamos aos participantes que o tema da oficina seria sexualidade, conforme eles haviam proposto nas urnas.

No aquecimento, enquanto se seguia a atividade de apresentação proposta, parte dos estudantes não participou, permanecendo sentados: alguns justificaram estar cansados, enquanto outros demonstraram indiferença pela atividade proposta. Porém aos poucos, percebemos o aumento do interesse no que ocorria na oficina e, gradualmente, eles passaram a interagir com os demais.

Iniciamos então a primeira atividade, a dinâmica do "Concordo ou não concordo?", que nos permitiu compreender os conhecimentos prévios dos estudantes sobre os temas abordados, bem como entender como pensavam e se relacionam com as diferentes proposições. Os estudantes foram unânimes e corretos ao avaliarem como corretas as afirmativas: (1) "Uma mulher pode engravidar mesmo que o homem ejacule fora dela" e (4) "Uma pessoa pode ter uma doença sexualmente transmissível sem ter nenhuma dor ou problema aparente".

Enquanto nas questões (2) "Anticoncepcional engorda e aumenta o risco de trombose" e (3) "É muito comum as mulheres engravidarem usando DIU", a maioria deles concordou com as afirmações, trazendo para a discussão relatos pessoais ou de conhecidos que passaram por tais 
situações. Já a questão (5) “A mulher pode saber exatamente o período do mês em que pode engravidar", causou grande polêmica. Dos 52 presentes, apenas três não concordaram com a afirmação. Pedimos que os estudantes apresentassem argumentos que os faziam concordar com a afirmativa e, dentre eles, expuseram conhecimentos sobre ciclo menstrual aprendidos em aulas de biologia e, especialmente as meninas, contaram sobre a existência de aplicativos de celular que preveem a data da ovulação da mulher, que muitas usam. A partir disso, elucidou-se sobre a possibilidade de prever uma "janela de fertilidade", sobre como ela pode variar entre as mulheres e como diferentes fatores, internos e externos, podem influenciá-la (BRASIL, 2013b).

As respostas e os argumentos utilizados mostraram a força das vivências pessoais na construção de opiniões e posicionamentos, comprovando a importância de valorizar e garantir espaços para que experiências pessoais sejam partilhadas. A PNEPS-SUS afirma que a construção compartilhada de saberes, enquanto processos entre pessoas e grupos diferentes, é fundamental para (des)(re)construir conhecimentos na perspectiva de entender e transformar as ações de saúde (BRASIL, 2005). Tal ideia é corroborada pelo conceito da salutogênese, pois é necessário que as informações científicas sejam apreendidas pelos sujeitos para que sejam articuladas com tais vivências, o que permite aos indivíduos reconhecerem suas capacidades de gestão e, assim, promoverem a capacidade de investimento, isto é, transformar o conhecimento em recursos protetivos de saúde enquanto mecanismo para superar situações adversas (LINDSTRÖM, 2018; SALCI et al., 2013),

A partir das discussões que as afirmações desencadearam, apresentamos dados científicos sobre os temas. Em relação aos anticoncepcionais, reforçou-se os fatores de riscos para trombose associados ao uso de contraceptivos hormonais e a efetividade do DIU, além de informarmos sobre como ter acesso ao DIU pelo Sistema Único de Saúde (SUS). Os estudantes mostram-se surpresos com as informações, até então desconhecidas. Além disso, reforçamos que apesar dos diferentes métodos contraceptivos, apenas os preservativos previnem IST.

Acreditamos que, a partir da discussão, contribuímos para o acesso a informações seguras, tanto no que tange às IST quanto ao planejamento reprodutivo, efetivando assim um dos pilares da Redução de Danos, que é instrumentalizar as pessoas para que sejam protagonistas de suas escolhas a partir de informações corretas e democratizar o acesso a insumos disponibilizados pelo SUS (BRASIL, 2013b), de modo a construir mecanismos individuais e 
coletivos de proteção à saúde e efetivar ações concretas por parte dos indivíduos, que podem incorporar tais discussões em suas realidades (SALCI et al., 2013).

$\mathrm{Na}$ segunda etapa da oficina, os estudantes foram divididos em pequenos grupos com média de oito pessoas cada, para os quais distribuímos as duas situações problemas que deveriam discutir.

Na situação 1, que se referia a planejamento familiar e métodos contraceptivos, os encaminhamentos apontados pelos estudantes foram que a mulher deveria buscar assistência médica para conhecer os diferentes tipos de métodos contraceptivos e, posteriormente, optar pelo mais adequado às suas necessidades e preferências, de modo a valorizar o processo de informação e o respeito à autonomia de cada sujeito (BRASIL, 2013a; 2012; 2005). Delimitaram também a existência de limites entre as opiniões do parceiro e o direito à escolha da mulher e foram enfáticos em reafirmarem a importância do uso do preservativo nas relações sexuais.

Ainda durante a discussão dessa situação, uma participante questionou sobre prevenção de IST para mulheres lésbicas, gerando espanto tanto nos colegas de turma quanto em nós, que conduzíamos a oficina, por não termos refletido sobre essa questão anteriormente. Diante disso, questionamos os estudantes se as IST também poderiam ser transmitidas durante o sexo entre mulheres, gerando divergência entre os estudantes. Esclarecemos então que, apesar de menos difundida a estatística sobre transmissibilidade quando comparado com o sexo heterossexual, as IST também podem ser transmitidas no sexo entre mulheres (ALMEIDA, 2009).

Questionamos quais métodos de proteção os estudantes conheciam e que poderiam ser adotados por essa população. Eles relataram o uso de preservativo masculino em dispositivos como dildos e vibradores para evitar a troca de secreção entre as parceiras durante o compartilhamento dos objetos, além do uso de dedeiras e protetores de língua, originalmente usados por fonoaudiólogos e dentistas, respectivamente. Também citaram o uso de plástico filme e preservativo masculino adaptados para a produção de barreira no sexo oral.

Apesar dos estudantes reconhecerem tais métodos, a literatura (BRASIL, 2013b) mostra que são pouco efetivos, tanto por serem pouco práticos de serem utilizados, quanto por não serem amplamente divulgados e incentivados por profissionais de saúde. 
A ausência de métodos próprios para o sexo entre mulheres evidencia as vulnerabilidades dessa população em relação a sua saúde, bem como a necessidade de criação de novas tecnologias para garantir o sexo seguro como um direito, considerando as diversidades individuais como a própria Política da Redução de Danos propõe (BRASIL, 2005).

Assim, legitimamos a demanda da estudante que nos alertou para a importância de se debater tal tema que, muitas vezes, é esquecido ou ignorado pelos profissionais de saúde, o que corrobora a invisibilidade das mulheres lésbicas e dificuldades do acesso dessa população a cuidados em saúde. Nessa situação, ficou clara a importância do conceito da salutogênese para potencializar a compreensão da Educação Popular em Saúde, pois, a partir da articulação entre informações e uma vivência individual foi possível problematizar a realidade em que a estudante está inserida e que representa a realidade da comunidade, permitindo pensar novas possibilidades de promoção de saúde e prevenção de doenças para o desenvolvimento de recursos protetivos para o empoderamento individual e coletivo (SALCI et al., 2013).

Na situação 2, que abordava a temática do assédio sexual, os estudantes majoritariamente afirmaram que, a partir do momento em que uma mulher diz não desejar algo, ela deve ser respeitada pelo parceiro, não podendo ter seu espaço invadido ou seu corpo violado. Entretanto, questionaram sobre a dificuldade do rapaz em interpretar os desejos da moça argumentando que "se ela apresentava desejo e curiosidade por que não gostaria de 'algo mais'?". Além disso, muitos questionaram o posicionamento da moça afirmando que "se ela não queria nada mais com ele, por que foi para um dos quartos da casa?" e mesmo que "se ela não quer nada, por que se colocou nessa situação? é claro que ela está querendo".

O grupo que culpabilizou a moça por seu comportamento era composto apenas por homens. Ao apresentarem a ideia, uma polêmica foi gerada com as mulheres participantes da atividade, que ficaram claramente incomodadas, contraponto as falas, reafirmando a autonomia das mulheres sobre seus próprios corpos e a necessidade de respeito para com suas escolhas e limites. Essa polêmica gerou muitas conversas paralelas e tumulto na sala, impossibilitando compreender e acompanhar claramente todas as discussões. Além disso, como o horário do final da aula já se aproximava e alguns estudantes demonstravam ansiedade por finalizar a atividade, a sala ficou bastante agitada.

Optamos por dar andamento à quarta etapa da oficina, espaço destinado para a turma dar um feedback sobre a atividade. No momento em que foi dada a palavra à turma, um estudante 
prontamente questionou: "masturbar muito faz mal?", evidenciando que a temática da sexualidade é de interesse dos estudantes e corroborando a compreensão de que a oficina não se propunha esgotar a discussão sobre o tema. Esse questionamento deixou clara a importância de assegurar espaços como esse de forma frequente.

Apesar da agitação dos estudantes, conseguimos ouvir alguns que se dispuseram a compartilhar com a turma suas opiniões sobre a oficina. Foram unânimes ao afirmar que o espaço proporcionado sanou dúvidas, permitiu mais debate e liberdade do que normalmente encontram na escola e que, de alguma forma, a oficina contribuiu para a vida deles. Reconheceram a importância de mais horários na grade curricular destinados a assuntos relacionados à sexualidade e reconheceram a importância do método utilizado.

\section{CONSIDERAÇÕES FINAIS}

A realização da oficina, desde o início, apresentou-se como um desafio, pois sexualidade é um tema de extrema relevância - principalmente na faixa etária trabalhada no projeto -, mas pouco abordado por professores e tutores, permeado de mitos e dúvidas e, sobretudo, delicado de ser discutido devido a crenças e valores morais. A nossa proposta foi abordar a temática de maneira que estimulasse o interesse e a participação dos acadêmicos e, ao mesmo tempo, trabalhasse a riqueza do tema. Porém, tínhamos 90 minutos e um grupo grande de estudantes.

O começo da oficina foi desafiador devido ao número maior de estudantes do que o pactuado com a escola, ou seja, para o que havíamos nos programado e que é preconizado nas teorias sobre metodologias ativas. Além disso, também tivemos dificuldade em função da agitação dos estudantes no início e foi preciso esforço para conseguir garantir atenção para explicar as atividades, fato que gerou desgaste para os interessados e nos mediadores.

Percebemos que o grande número de participantes na oficina prejudicou a dinâmica pelo fato de alguns não estarem interessados e dispersarem, facilmente prejudicando os demais. Além disso, contribuiu para aumentar a inibição de alguns estudantes. $\mathrm{O}$ envolvimento durante a oficina em grupos reduzidos (até 15 pessoas) foi maior, pois facilitou o diálogo, bem como a mediação dos debates dentro dos pequenos grupos, em concordância com o que aponta a literatura (FREIRE, 1996).

Em contrapartida, ficamos num impasse quando tentávamos manejar a agitação dos estudantes, pois não desejávamos repetir o modelo punitivista com que são acostumados no 
ambiente escolar. As oficinas são propostas com a lógica de permitir autonomia aos estudantes, portanto não fazia sentido impor uma atividade, ou pior, aplicar sansões aos que não quisessem participar.

Outro impasse, além do exposto acima, foi o fato da escola exigir a participação dos estudantes na oficina devido ao horário obrigatório a ser cumprido na grade curricular. Porém, tentamos, durante as atividades, deixar quem não desejasse participar ficar dentro da sala, porém realizando atividades que fossem do próprio interesse sem, contudo, atrapalhar a dinâmica proposta para o restante do grupo.

Outro ponto a ser destacado é a inexperiência dos estudantes quanto à metodologia participativa. Como são acostumados a um modelo impositivo e que oferece pouca autonomia, acreditamos que, ao proporcionarmos um espaço como foi o da oficina, muitos não souberam lidar com a liberdade oferecida, justificando a agitação.

Apesar das dificuldades, avaliamos que conseguimos envolver a maior parte dos estudantes da sala nas atividades propostas, permitindo a participação e exposição de ideias diversas, surgimento de polêmicas e informações enriquecedoras a todo o grupo. Promovemos debates, espaços de fala, de crítica e o conhecimento para o autocuidado. Entendemos que as experiências vividas são fundamentais no trabalho de uma oficina em que se pretende discutir temas relacionados à saúde já que as dúvidas e conhecimentos apresentados por cada participante fomentam debate e aprendizado conjunto.

Os estudantes vivenciaram uma metodologia distinta da que estão acostumados no ensino tradicional, bem como experimentaram a abordagem da redução de danos e da educação em saúde. A experiência de autonomia e troca de conhecimentos durante o trabalho possibilitou o desenvolvimento de capacidades individuais e coletivas para a geração de recursos protetivos de saúde e para o não adoecimento, como respaldado pela PNEPS-SUS, revelando as potencialidades da Educação Popular em Saúde. Assim, percebemos a importância da abordagem de temáticas relevantes à formação dos jovens nas escolas, sobretudo, trabalhar a autonomia por meio de atividades com metodologias participativas. 


\section{REFERÊNCIAS}

ABREU, P. H. B.; ALONZO, H. G. A. Salutogênese - camponês a camponês: uma metodologia para promoção da saúde de populações expostas a agrotóxicos. Saúde em Debate, Rio de Janeiro, v. 42, p. 261-274, 2018. Doi: 10.1590/0103-11042018s421.

ALMEIDA, G. Argumentos em torno da possibilidade de infecção por DST e Aids entre mulheres que se autodefinem como lésbicas. Physis, Rio de Janeiro, v. 19, n. 2, p. 301331, 2009. Doi: 10.1590/S0103-73312009000200004.

BRASIL. Ministério da Educação. Resolução n ${ }^{\text {3 3, de }} 20$ de junho de 2014. Institui Diretrizes Curriculares Nacionais do Curso de Graduação em Medicina. Diário Oficial da União, Brasília, DF, 23 de junho de 2014.

BRASIL. Ministério da Saúde. Departamento de Atenção Básica. Política Nacional de Atenção Básica. Brasília: Ministério da Saúde, 2012.

BRASIL. Ministério da Saúde. Secretaria de Vigilância em Saúde. Saúde e prevenção nas escolas: guia para a formação de profissionais de saúde e de educação. Brasília: Ministério da Saúde, 2006.

BRASIL. Ministério da Saúde. Gabinete do Ministro. Portaria no 2.761, de 19 de novembro de 2013. Institui a Política Nacional de Educação Popular em Saúde no Âmbito do Sistema Único de Saúde (PNEPSSUS). Diário Oficial da União, Brasília, DF, 20 nov. $2013 a$.

BRASIL. Ministério da Saúde. Gabinete do Ministro. Portaria n ${ }^{\circ} 2.194$, de 14 de outubro de 2004. Redefine e amplia a atenção integral para usuários de álcool e outras drogas, no âmbito do Sistema Único de Saúde (SUS). Diário Oficial da União, Brasília, DF, 14 de outubro de 2014.

BRASIL. Ministério da Saúde. Secretaria de Atenção à Saúde. Departamento de Atenção Básica. Saúde sexual e saúde reprodutiva. Brasília: Ministério da Saúde, 2013b.

CROMACK, L. (org). Oficina de ideias: manual de dinâmicas. Rio de Janeiro, 2003.

FERREIRA, M. S. Agite antes de usar... A Promoção da saúde em programas brasileiros de promoção da atividade física: o caso do Agita São Paulo. 2008. 264 f. Tese (Doutorado em Ciências) - Escola Nacional de Saúde Pública Sérgio Arouca, Fundação Oswaldo Cruz, Rio de Janeiro. 2008.

FRAGA, A. B. et al. Curso de extensão em promoção de saúde para gestores do SUS com enfoque no Programa Academia da Saúde. Brasília: Ministério da Saúde; Secretaria de Vigilância em Saúde; CEAD/UnB, 2013.

FREIRE, P. Pedagogia da autonomia: saberes necessários à prática educativa. 25. ed. São Paulo: Paz e Terra, 1996.

LINDSTRÖM, B. Workshop salutogenesis and the future of health promotion and public health. Scandinavian Journal of Public Health, v. 46, n. 20, p. 94-98, 2018. Doi: $10.1177 / 1403494817743902$. 
KUMAR, S.; PREETHA, G. S. Health promotion: an effective tool for global health. Indian J Community Med, v. 37, n. 1, p. 5-12, 2012. Doi: 10.4103/0970-0218.94009.

PEDROSA, J. I. S. Educação Popular e promoção da saúde: bases para o desenvolvimento da escola que produz saúde. In: BRASIL. Ministério da Saúde. Escolas promotoras de saúde: experiências do Brasil. Brasília: Ministério da Saúde; Organização Pan-Americana da Saúde, 2006. p. 41-48.

RAIMONDI, G. A. et al. Intersetorialidade e educação popular em saúde: no SUS com as escolas e nas escolas com o SUS. Revista Brasileira de Educação Médica, Brasília, v. 42, n. 2, p. 73-78, 2018. Doi: 10.1590/1981-52712015v42n2rb20170043.

SALCI, M. A. et al. Educação em saúde e suas perspectivas teóricas: algumas reflexões. Texto Contexto Enfermagem, Florianópolis, v. 22, n. 1, p. 224-230, $2013 . \quad$ Doi: 10.1590/S0104-07072013000100027.

VALADÃO, M. M. Saúde na Escola: um campo em busca de espaço na agenda intersetorial. 2004. 154 f. Tese (Doutorado em Serviços de Saúde) - Departamento de Prática de Saúde Pública, Universidade de São Paulo, São Paulo. 2004.

Submetido em 21 de agosto de 2019.

Aprovado em $1^{\circ}$ de outubro de 2019. 